\title{
Evidence for exchange bias coupling at the perovskite/brownmillerite interface in spontaneously stabilized $\mathrm{SrCoO}_{3-\delta} / \mathrm{SrCoO}_{2.5}$ bilayers
}

\author{
B. C. Behera, ${ }^{1, *}$ Subhadip Jana,${ }^{1,2}$ Shwetha G. Bhat,${ }^{3}$ N. Gauquelin,${ }^{4}$ G. Tripathy,${ }^{1,2}$ P. S. Anil Kumar, ${ }^{3}$ and D. Samal ${ }^{1,2,}$ \\ ${ }^{1}$ Institute of Physics, Sachivalaya Marg, Bhubaneswar-751005, India \\ ${ }^{2}$ Homi Bhabha National Institute, AnushaktiNagar, Mumbai-400085, India \\ ${ }^{3}$ Department of Physics, Indian Institute of Science Bangalore-560012, India \\ ${ }^{4}$ EMAT, University of Antwerp - Groenenborgerlaan 171, 2020 Antwerp, Belgium
}

(Received 24 August 2018; revised manuscript received 11 November 2018; published 23 January 2019)

\begin{abstract}
Interface effect in complex oxide thin-film heterostructures lies at the vanguard of current research to design technologically relevant functionality and explore emergent physical phenomena. While most of the previous works focus on the perovskite/perovskite heterostructures, the study of perovskite/brownmillerite interfaces remains in its infancy. Here, we investigate spontaneously stabilized perovskite-ferromagnet $\left(\mathrm{SrCoO}_{3-\delta}\right)$ /brownmillerite-antiferromagnet $\left(\mathrm{SrCoO}_{2.5}\right)$ bilayer with $T_{\mathrm{N}}>T_{\mathrm{C}}$ and discover an unconventional interfacial magnetic exchange bias effect. From magnetometry investigations, it is rationalized that the observed effect stems from the interfacial ferromagnet/antiferromagnet coupling. The possibility for coupled ferromagnet/spin-glass interface engendering such effect is ruled out. Strikingly, a finite coercive field persists in the paramagnetic state of $\mathrm{SrCoO}_{3-\delta}$, whereas the exchange bias field vanishes at $T_{\mathrm{C}}$. We conjecture the observed effect to be due to the effective external quenched staggered field provided by the antiferromagnetic layer for the ferromagnetic spins at the interface. Our results not only unveil a paradigm to tailor the interfacial magnetic properties in oxide heterostructures without altering the cations at the interface, but also provide a purview to delve into the fundamental aspects of exchange bias in such unusual systems, paving a big step forward in thin-film magnetism.
\end{abstract}

DOI: 10.1103/PhysRevB.99.024425

\section{INTRODUCTION}

The investigation on interfacial magnetic effects in transition-metal-oxide based thin-film heterostructures has sparked unprecedented scientific developments and is pursued intensively because of its technological promise for the nextgeneration nanoscaled magnetic devices [1]. A precise control and tuning of interfacial magnetic properties in thin-film heterostructures is crucial for engendering exotic functionalities which are highly relevant for technological applications such as magnetic-field sensors, memories, or magnetic recording read heads [2-4]. A great deal of attention in this regard is focused on the effect called "exchange bias" that occurs due to interfacial magnetic exchange coupling in a coupled ferromagnetic/antiferromagnetic (FM/AF) system [5,6]. This effect is widely maneuvered for the design and operation of spin valve based magnetic read heads and sensors. The macroscopic hallmark of magnetic exchange bias effect (MEBE) is the unidirectional shift of the $M(H)$ loop along the field axis [Fig. 1(c)], and enhancement of coercivity. Typically, a bilayer consisting of a FM and an AF [with the Curie temperature $\left(T_{\mathrm{C}}\right)$ of FM greater than the Néel temperature $\left(T_{\mathrm{N}}\right)$ of $\left.\mathrm{AF}\right]$ when cooled in a static magnetic field across the $T_{\mathrm{N}}$, a unidirectional exchange anisotropy field gets locked in and gives rise to exchange bias effect that stabilizes the

\footnotetext{
*bcbehera@iopb.res.in

$\dagger$ dsamal@iopb.res.in
}

orientation of the ferromagnetic layer $[2,7,8]$. Such systems exhibit magnetic properties that markedly differ from their constituents. Although exchange bias related phenomena in FM/AF coupled system are studied extensively, its inherent mechanism has not been completely understood because it is always hard to directly observe and manipulate the spin structure at the interface.

Hitherto, MEBE has been observed in numerous metaloxides FM/AF coupled systems (e.g., $\mathrm{La}_{0.67} \mathrm{Sr}_{0.37} \mathrm{MnO}_{3}$ / $\mathrm{SrMnO}_{3}$ [9], $\mathrm{La}_{0.67} \mathrm{Ca}_{0.37} \mathrm{MnO}_{3} / \mathrm{SrMnO}_{3}$ [10], $\mathrm{La}_{0.67} \mathrm{Sr}_{0.37}$ $\mathrm{MnO}_{3} / \mathrm{BiFeO}_{3}$ [11], $\mathrm{La}_{0.67} \mathrm{Sr}_{0.37} \mathrm{MnO}_{3} / \mathrm{TbMnO}_{3}$ [12]) with the $T_{\mathrm{N}}$ of the accompanying $\mathrm{AF}$ always being lower than the $T_{\mathrm{C}}$ of the FM. Thus, it has been generally accepted that the $T_{\mathrm{C}}>T_{\mathrm{N}}$ criterion is a prerequisite for establishing exchange bias effect at the FM/AF interface. Indeed, all theoretical models have virtually relied on the assumption of the $T_{\mathrm{C}}>T_{\mathrm{N}}$ criterion to delve into the mechanism for interfacial coupling $[5,6,13,14]$. Regardless of the long orthodox belief of the $T_{\mathrm{C}}>T_{\mathrm{N}}$ criterion, a few remarkable experimental observations manifesting MEBE are reported in FM/AF bilayer systems with $T_{\mathrm{N}}>T_{\mathrm{C}}$. For instance, $\mathrm{Wu}$ and Chien observed exchange coupling phenomena in FM/AF bilayers of $a-\left(\mathrm{Fe}_{0.1} \mathrm{Ni}_{0.9}\right)_{80} \mathrm{~B}_{20}\left(T_{\mathrm{C}} \sim 240 \mathrm{~K}\right) / \mathrm{CoO}\left(T_{\mathrm{N}}=291 \mathrm{~K}\right)$ and $a-\mathrm{Fe}_{4} \mathrm{Ni}_{76} \mathrm{~B}_{20}\left(T_{\mathrm{C}} \sim 150 \mathrm{~K}\right) / \mathrm{CoO}\left(T_{\mathrm{N}} \sim 291 \mathrm{~K}\right)$, respectively $[15,16]$. Similar effect was also observed in the $\mathrm{MnO}$ (antiferromagnet) $/ \mathrm{Mn}_{3} \mathrm{O}_{4}$ (ferrimagnet) core/shell structure [17]. Here we explore such effect in a perovskite $\left(\mathrm{SrCoO}_{3-\delta}\right)$ /brownmillerite $\left(\mathrm{SrCoO}_{2.5}\right)$ thin-film interface, wherein bulk $\mathrm{SrCoO}_{3}$ hosts a metallic ferromagnetic state 


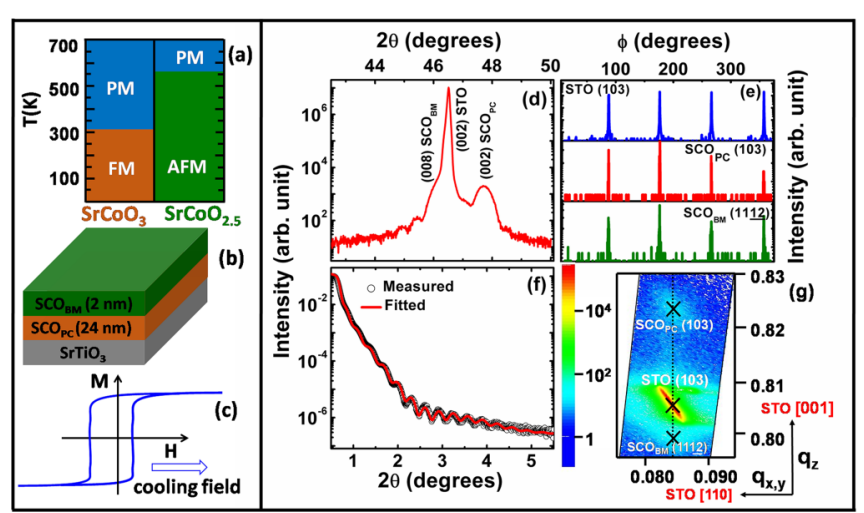

FIG. 1. Left panel: (a) schematic representation of magnetic ordering temperature for ferromagnetic $\mathrm{SrCoO}_{3}$ and antiferromagnetic $\mathrm{SrCoO}_{2.5}$; (b) layout of the designed $\left[\operatorname{SCO}_{\mathrm{PC}}(\sim 24 \mathrm{~nm}) / \mathrm{SCO}_{\mathrm{BM}}(\sim 2 \mathrm{~nm})\right]$ bilayer on STO; (c) schematic MHL representing MEBE under positive field cooling. Right panel: (d) $\theta-2 \theta$ x-ray diffraction pattern; (e) $\phi$ scan along the asymmetric planes of $\mathrm{STO}(103), \mathrm{SCO}_{\mathrm{PC}}(103), \mathrm{SCO}_{\mathrm{BM}}(11 \underline{12})$; (f) measured and fitted x-ray reflectivity, and (g) off-specular reciprocal space mapping around $\operatorname{STO}(103)$ of $\left[\operatorname{SCO}_{\mathrm{PC}}(\sim 24 \mathrm{~nm}) / \operatorname{SCO}_{\mathrm{BM}}(\sim 2 \mathrm{~nm})\right]$ bilayer.

with $T_{\mathrm{C}}$ in the range $280-305 \mathrm{~K}[18,19]$ and $\mathrm{SrCoO}_{2.5}$ exhibits an insulating antiferromagnetic state with $T_{\mathrm{N}} \sim 570 \mathrm{~K}[20,21]$ as sketched in Fig. 1(a).

$\mathrm{SrCoO}_{x}$ exhibits highly contrasting structural, electronic, and magnetic property depending on the Co oxidation state, which can be manipulated by controlling the oxygen stoichiometry [19,22-26]. Brownmillerite $\mathrm{SrCoO}_{2.5}\left(\mathrm{SCO}_{\mathrm{BM}}\right)$ derives its structure from the perovskite $\mathrm{SrCoO}_{3}\left(\mathrm{SCO}_{\mathrm{PC}}\right)$ through the removal of $1 / 6$ of oxygen atoms such that alternating oxygen octahedral and tetrahedral are stacked together [18]. While bulk $\mathrm{SCO}_{\mathrm{BM}}$ is readily synthesized under ambient condition, $\mathrm{SCO}_{\mathrm{PC}}$ limits its synthesis to extremely high pressure, due to relatively large thermodynamic energy barrier for the formation of perovskite phase involving $\mathrm{Co}^{4+}$ ions. However, recent studies reveal the epitaxial stabilization of single-crystalline $\mathrm{SCO}_{\mathrm{PC}}$ thin films via topotactic phase transformation under high oxidizing condition [22-24]. Manipulating the oxygen sublattice in complex oxide thin-film heterostructure/interface offers a promising avenue to look for fascinating functionality. Here we report the fabrication of FM-perovskite $\left(\mathrm{SrCoO}_{3-\delta}\right) / \mathrm{AF}$-brownmillerite $\left(\mathrm{SrCoO}_{2.5}\right)$ natural bilayer by pulsed-laser epitaxy and demonstrate the evidence for unconventional exchange bias with $T_{\mathrm{N}}>T_{\mathrm{C}}$. The term "natural bilayer" is coined categorically to emphasize that an interface involving perovskite- $\mathrm{SrCoO}_{3}$ and brownmillerite- $\mathrm{SrCoO}_{2.5}$ is formed spontaneously as shown in Fig. 1(b). The perovskite/brownmillerite interfaces are expected to host emergent interfacial phenomena due to lattice symmetry mismatch, but remain scantly explored [27,28]. A recent study by Zhang et al. found robust perpendicular magnetic anisotropy for $\mathrm{La}_{2 / 3} \mathrm{Sr}_{1 / 3} \mathrm{MnO}_{3} / \mathrm{LaCoO}_{2.5}$ [27]. The present revelation for the spontaneous stabilization of perovskite/brownmillerite $\left(\mathrm{SCO}_{\mathrm{PC}} / \mathrm{SCO}_{\mathrm{BM}}\right)$ interface without altering the cations uniquely provides an ideal system to investigate the interfacial phenomena. In particular, the realization of unusual MEBE in (FM-SCO $\left.{ }_{\mathrm{PC}} / \mathrm{AF}-\mathrm{SCO}_{\mathrm{BM}}\right)$ bilayer in this study constitutes a fundamental step to broaden the search to a greater variety of FM/AF bilayer with $T_{\mathrm{N}}>T_{\mathrm{C}}$ exhibiting exchange bias related phenomena that will have relevant implications in technological applications.

\section{EXPERIMENTAL RESULTS AND DISCUSSION}

High-quality epitaxial natural bilayers consisting of $\mathrm{SCO}_{\mathrm{PC}}$ and $\mathrm{SCO}_{\mathrm{BM}}$ with varying thickness were grown on the single-crystalline STO(001) substrates ( $a=3.905 \AA)$ using pulsed-laser deposition. Details of deposition method are described in Supplemental Material [29]. SCOPC exhibits a typical $\mathrm{ABO}_{3}$ perovskite structure with cubic $P m \underline{3} m$ symmetry $(a=3.829 \AA)$ while $\mathrm{SCO}_{\mathrm{BM}}$ forms an orthorhombic Ima2 symmetry with $a=5.574 \AA, b=5.469 \AA$, and $c=15.745 \AA$ [30]. In a pseudotetragonal setting, the lattice parameters of $\mathrm{SCO}_{\mathrm{BM}}$ can be expressed as $a_{t}=3.905 \AA$ and $c_{t}=3.936 \AA$, where $a_{t}$ and $c_{\mathrm{t}}$ are the lattice parameters of the reduced pseudotetragonal representation of Ima 2 symmetry. We present the results on two representative bilayers: $\left[\operatorname{SCO}_{\mathrm{PC}}(\sim 24 \mathrm{~nm}) / \operatorname{SCO}_{\mathrm{BM}}(\sim 2 \mathrm{~nm})\right]$ and $\left[\operatorname{SCO}_{\mathrm{PC}}(\sim 20 \mathrm{~nm}) / \operatorname{SCO}_{\mathrm{BM}}(\sim 2 \mathrm{~nm})\right]$. The structural characterization is carried out using a high-resolution X-ray diffractometer (Rigaku, Smart Lab). The $\theta-2 \theta$ XRD pattern of bilayers is found to be oriented along (001) as shown in Fig. 1(d). The full range of $\theta-2 \theta$ XRD pattern is shown in Supplemental Material (Fig. S1). To determine the thickness of individual layers and the stacking order, $x$-ray reflectivity (XRR) measurement is performed and the corresponding data were fitted using GLOBALFIT software of Rigaku [Fig. 1(f) and Fig. S2]. The results from XRR fitting inferred the stacking order to follow $\left[\mathrm{STO} / \mathrm{SCO}_{\mathrm{PC}} / \mathrm{SCO}_{\mathrm{BM}}\right]$ type, indicating the possible oxygen vacancies in proximity to the surface could drive the top layer into $\mathrm{BM}$ phase in the bilayers. The reverse order of stacking such as $\left[\mathrm{STO} / \mathrm{SCO}_{\mathrm{BM}} / \mathrm{SCO}_{\mathrm{PC}}\right]$ was ruled out as the latter gave an inadequate fit to the XRR data. From XRR fitting, the average surface roughness of the bilayer was found to be $\sim 0.5 \mathrm{~nm}$, indicating an atomically smooth surface. The observation of Kiessig fringes and wellpronounced Laue oscillations in the X-ray spectra are also a clear indication of superior quality of the bilayers. The out-of-plane lattice parameters " $c$ " calculated from (001) peak positions for $\left[\operatorname{SCO}_{\mathrm{PC}}(\sim 24 \mathrm{~nm}) / \mathrm{SCO}_{\mathrm{BM}}(\sim 2 \mathrm{~nm})\right]$ bilayer are 3.809 and $3.920 \AA$ for $\mathrm{SCO}_{\mathrm{PC}}$ and $\mathrm{SCO}_{\mathrm{BM}}$, respectively. By considering an idealistic fully in-plane strained case with $a=b=3.905 \AA$ (lattice parameter of STO) and assuming the unit-cell volume conservation, one would naively expect the $c$-axis lattice parameter of $\mathrm{SCO}_{\mathrm{PC}}$ to be $\sim 3.681 \AA$. The observed increase in $c$-axis lattice parameter could either be due to oxygen deficiency or strain-induced octahedral distortion. Although it is really hard to accurately determine the exact amount of oxygen content from XRD in the present study, a naive estimation of oxygen content can be inferred from magnetization study in the succeeding section. To elucidate the in-plane epitaxial relationship in the bilayers, we performed the $\phi$ scan and reciprocal space mapping with respect to STO (103), $\operatorname{SCO}_{\mathrm{PC}}(103)$, and $\operatorname{SCO}_{\mathrm{BM}}(11 \underline{12})$ planes. Four equally spaced distinct peaks with a relative separation of $90^{\circ}$ 


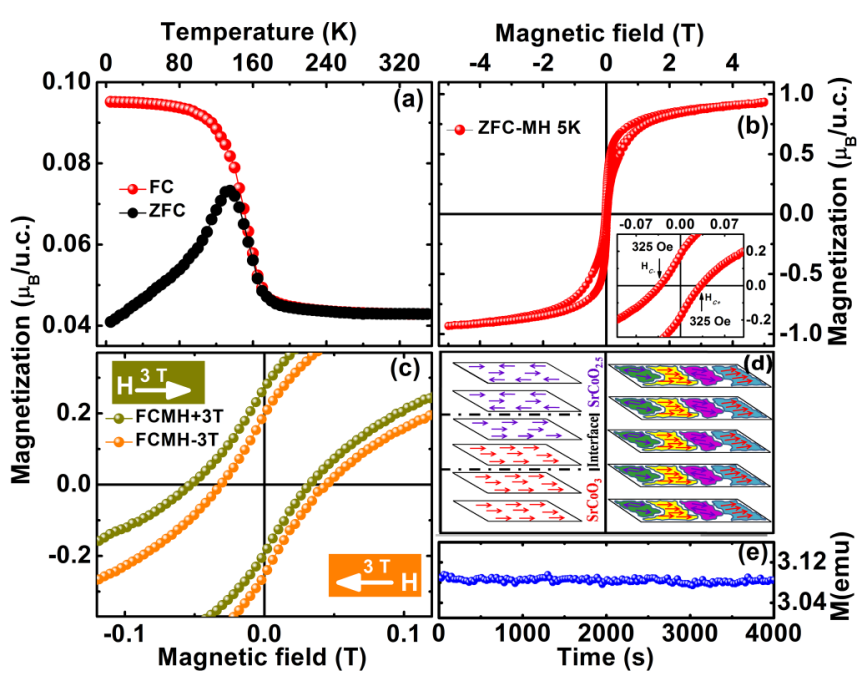

FIG. 2. (a) Temperature-dependent zero-field-cooled and fieldcooled magnetization. (b) $M(H)$ loop at $5 \mathrm{~K}$ after zero-field cooling from room temperature [the inset shows the enlarged view of $M(H)$ loop indicating symmetric coercive field on positive and negative field axis]. (c) $M(H)$ loops at $5 \mathrm{~K}$ after field cooling from $350 \mathrm{~K}$ in a $+3-\mathrm{T}$ field (dark yellow circles) and in a $-3-\mathrm{T}$ field (orange circles). (d) Schematic representation of two possible growth structures, and (e) the thermoremnant magnetization of $\left[\mathrm{SCO}_{\mathrm{PC}}(\sim 24 \mathrm{~nm}) / \mathrm{SCO}_{\mathrm{BM}}(\sim 2 \mathrm{~nm})\right]$ bilayer.

(fourfold symmetry) were observed in the $\phi$ scans, suggesting the cube-on-cube epitaxial growth of the constituent layers on STO, i.e., [100]STO $\|$ [100]SCO $\mathrm{PC}_{\|} \|[100] \mathrm{SCO}_{\mathrm{BM}}$. From reciprocal mapping, it was observed that the diffraction peaks associated with STO and the constituting layers lie at the same $q_{x}$ value, and thus indicate the bilayer to be completely strained with respect to the underlying substrate [Fig. 1(g)]. In essence, our extensive structural investigation elucidates the occurrence of strained epitaxial $\mathrm{SrCoO}_{3-\delta} / \mathrm{SrCoO}_{2.5}$ natural bilayer on STO.

Magnetic measurements [magnetization $(M)$ versus temperature and field $(H)$ in zero-field-cooled (ZFC) and fieldcooled (FC) mode] were performed by a superconducting quantum interface device based magnetometer (Quantum Design SQUID-VSM). The magnetic field was set to zero in an oscillation mode to reduce the residual field before measurements. The residual field was further calibrated by a reference Pd sample that shows a negligible value (see Supplemental Material [29]). Systematic analysis of the magnetic data after correcting for the diamagnetic substrate contribution was carried out to determine the coercive and exchange bias field (see Supplemental Material). In Fig. 2(a), we show the FC and ZFC temperature-dependent magnetization $M(T)$ for $\left[\operatorname{SCO}_{\mathrm{PC}}(\sim 24 \mathrm{~nm}) / \mathrm{SCO}_{\mathrm{BM}}(\sim 2 \mathrm{~nm})\right]$ bilayer [hereafter abbreviated as $\left(\mathrm{SCO}_{\mathrm{PC}} / \mathrm{SCO}_{\mathrm{BM}}\right)$ for the sake of brevity unless explicitly clarified] with a field of 100 Oe applied along the in-plane direction of (001) STO. A ferromagneticlike order is apparent from $M(T)$ with an onset of transition at $\sim 175 \mathrm{~K}$. The value of observed $T_{\mathrm{C}} \sim 175 \mathrm{~K}$ is found to be lower than that of its $\mathrm{SCO}_{\mathrm{PC}}$ bulk counterpart in which the $T_{\mathrm{C}}$ ranges from 280 to $305 \mathrm{~K}[18,19]$. The diminished $T_{\mathrm{C}}$ in thin films could be attributed to strain and finite size $\left[T_{\mathrm{C}}(\infty)-T_{\mathrm{C}}(t)\right] / T_{\mathrm{C}}(\infty)=$ $(c / t)^{\lambda}$, where $T c(\infty)$ is the Curie temperature in the bulk limit, $T c(t)$ is the Curie temperature of the films according to their thickness, $c$ is related to spin-correlation length, $t$ is the film thickness, and $\lambda$ is the critical shift exponent) effects [22,23,31-33]. Indeed, it has been reported that as the degree of substrate-induced tensile strain increases from $\left.0.9 \%\left[\left(\mathrm{LaAlO}_{3}\right)_{0.3^{-}}\left(\mathrm{SrAl}_{0.5} \mathrm{Ta}_{0.5} \mathrm{O}_{3}\right)_{0.7}\right)\right]$ to $1.8 \%$ (STO), the ferromagnetic transition temperature reduces from $\sim 250$ to $\sim 200 \mathrm{~K}$ [22,23]. Interestingly, we observe a relatively higher $T_{\mathrm{C}}(\sim 188 \mathrm{~K})$ when the thickness of the $\mathrm{SCO}_{\mathrm{PC}}$ layer increases to $\sim 31 \mathrm{~nm}$, i.e., for $\left[\operatorname{SCO}_{\mathrm{PC}}(\sim 31 \mathrm{~nm}) / \operatorname{SCO}_{\mathrm{BM}}(\sim 4 \mathrm{~nm})\right]$ bilayer and thus justifies the role of finite-size effect on observed lower $T_{\mathrm{C}} \sim 175 \mathrm{~K}$ in $\left[\operatorname{SCO}_{\mathrm{PC}}(\sim 24 \mathrm{~nm}) / \operatorname{SCO}_{\mathrm{BM}}(\sim 2 \mathrm{~nm})\right]$ bilayer. Besides strain and dimensional effect, the $T_{\mathrm{C}}$ in $\mathrm{SCO}_{\mathrm{PC}}$ is also prone to oxygen deficiency $[19,23,34]$. It has to be mentioned that the work by Callori et al. on $\mathrm{SrCoO}_{3-\delta}$ thin films $(40 \mathrm{~nm})$ grown on STO reported a $T_{\mathrm{C}} \sim 170 \mathrm{~K}$ with $\delta \leqslant$ 0.18 [35]. Considering and comparing the $T_{\mathrm{C}}$ as a reference to naively determine oxygen stoichiometry, we believe the oxygen content of $\mathrm{SCO}_{\mathrm{PC}}$ layer in the present study is close to optimal doping with $\delta<0.18$. Besides, the XRD pattern in Fig. 1 does not reveal any noticeable oxygen vacancy superstructure, which also indicates that $\mathrm{SCO}_{\mathrm{PC}}$ film is close to optimal doping. Interestingly, we observe a finite magnetization above $T_{\mathrm{C}}$ unlike a conventional FM-paramagnetic (PM) transition [Fig. 2(a)]. Similar features are also observed in the $\left[\operatorname{SCO}_{\mathrm{PC}}(\sim 20 \mathrm{~nm}) / \mathrm{SCO}_{\mathrm{BM}}(\sim 2 \mathrm{~nm})\right]$ bilayer (see Supplemental Material).The finite magnetization above $T_{\mathrm{C}}$ seen in these bilayers could be attributed to staggered effective magnetic field with a nonzero average provided by the spins at the interfacial layer. In order to give a general baseline, we present and illustrate a comparative $\mathrm{M}$ vs $T$ plot for pure $\mathrm{SCO}_{\mathrm{PC}}$ and $\mathrm{SCO}_{\mathrm{BM}}$ single layers, as well as $\left(\mathrm{SCO}_{\mathrm{PC}} / \mathrm{SCO}_{\mathrm{BM}}\right)$ bilayer (see Fig. S6). Albeit the direct probe to AF transition $\mathrm{SrCoO}_{3-\delta} / \mathrm{SrCoO}_{2.5}$ bilayers is constrained because of experimental limitation to access high-temperature magnetic measurements, sufficient evidences based on magnetometry measurements are inferred in the succeeding section to warrant the existence of antiferromagnetic $\mathrm{SCO}_{\mathrm{BM}}$ layer conjointly with ferromagnetic $\mathrm{SCO}_{\mathrm{PC}}$ layer. To substantiate the existence of antiferromagnetic character in $\left(\mathrm{SCO}_{\mathrm{PC}} / \mathrm{SCO}_{\mathrm{BM}}\right)$ bilayer, we present the ZFC $M(H)$ plot at $5 \mathrm{~K}$. A few noteworthy points can be identified from the $M(H)$ loop: (a) it shows a ferromagneticlike hysteresis loop; however, the loop gets significantly constricted near zero-field region; (b) magnetization increases monotonically with applied magnetic field with no saturation even up to $5 \mathrm{~T}$; (c) the magnetization value obtained at $5 \mathrm{~T}$ is about $1 \mu_{\mathrm{B}}$ /unit cell, which is significantly smaller than values reported for the single crystal $\left(\sim 2.5 \mu_{\mathrm{B}} / \mathrm{Co}^{4+}\right.$ [18]) and epitaxial thin films of only $\mathrm{SCO}_{\mathrm{PC}}$ [22]. All the above observations in the ZFC $M(H)$ loop indicate towards the possible existence of FM/AF exchange coupling at the interface in $\left(\mathrm{SCO}_{\mathrm{PC}} / \mathrm{SCO}_{\mathrm{BM}}\right)$ bilayer.

The interfacial FM/AF exchange coupling is widely probed by MEBE that shows a displacement of the ferromagnetic hysteresis loop along the magnetic-field axis. Further, the direction of the loop shift reverses when the cooling field is reversed. Followed by the indication for interfacial magnetic 
exchange coupling from the ZFC $M(H)$ plots, we measured the field-cooled $M(H)$ loop to examine the possible emergence of MEBE. In-plane magnetic hysteresis loops measured at $5 \mathrm{~K}$ after field cooling from 350 to $5 \mathrm{~K}$ in applied fields of +3 and $-3 \mathrm{~T}$ are shown in Fig. 2(c). Interestingly, the center of magnetic hysteresis loop (MHL) was observed to shift by $\sim 105$ Oe along the $-v e$ and $+v e$ side of field axes for applied fields of +3 and $-3 \mathrm{~T}$, respectively, manifesting the sign of negative MEBE. Similar exchange bias effect was also observed for $\left[\operatorname{SCO}_{\mathrm{PC}}(\sim 20 \mathrm{~nm}) / \operatorname{SCO}_{\mathrm{BM}}(\sim 2 \mathrm{~nm})\right]$ and $\left[\operatorname{SCO}_{\mathrm{PC}}(\sim 31 \mathrm{~nm}) / \mathrm{SCO}_{\mathrm{BM}}(\sim 4 \mathrm{~nm})\right]$ bilayers as illustrated in Figs. S10 and S11, which suggests the observed effect is generic to spontaneously stabilized $\left[\mathrm{SCO}_{\mathrm{PC}} / \mathrm{SCO}_{\mathrm{BM}}\right]$ interface. The observed effect in the present case is reminiscent of the recent work by Migliorini et al. that reported the spontaneous exchange bias formation driven by a structural phase transition associated with $\mathrm{IrMn}$ in $\mathrm{IrMn} / \mathrm{FeCo}$ bilayer [36]. To shed light on the possible microscopic origin of the observed MEBE in the present case, we consider two possible structures as shown schematically in Fig. 2(d). In one case, we consider layered-by-layered structure with a sharp FM/AF interface, and in the other case we consider a random mixture of FM and AF clusters that could give rise to a spin-glass (SG)-like phase at the interfaces due to intercluster interaction. Although the manifestation of MEBE is commonly observed when a FM is in contact with an AF, there are instances of observing such effect in FM/SG coupled systems [37]. If the observed MEBE was due to FM-SG coupling, it would be natural to expect time-dependent slow dynamics response in magnetization since SG states are intrinsic to numerous metastable states. In a SG system, time decay of the thermoremnant magnetization (TRM) generally follows a logarithmic trend [38]. As shown in Fig. 2(e), the TRM for $\left(\mathrm{SCO}_{\mathrm{PC}} / \mathrm{SCO}_{\mathrm{BM}}\right)$ bilayer in contrast remains almost constant over four decades of observation time (see Supplemental Material for the protocol used for TRM measurement). Thus, we rule out the possibility of any coupled FM/SG interface resulting in $\mathrm{MEBE}$ in $\left(\mathrm{SCO}_{\mathrm{PC}} / \mathrm{SCO}_{\mathrm{BM}}\right)$ bilayer.

Further, to elucidate about the temperature dependence of MEBE, we measured field-cooled $M(H)$ loops at various temperatures (Fig. S9). The measured loops revealed a systematic change in asymmetry and coercivity. For every successive measurement at each fixed temperature, the sample was field cooled from $350 \mathrm{~K}$ with an applied field of $3 \mathrm{~T}$. To demonstrate the MEBE more clearly, we have plotted the MHL using the inversion method [39], in which $M$ and $H$ of the reversing part of the original loop are multiplied by -1 and the modified loop so-called "inverted loop" is presented in Fig. 3. In the inverted loop, the $H_{C-}$ value of the original loop gets shifted to positive field side, and thus we can see the difference between $H_{C+}$ of original loop and $H_{C-}$ of inverted loop with clarity. Enlarged curves of original and inverted hysteresis loops are shown in Figs. 3(a)-3(i). It is evident that MEBE gradually becomes weaker with increasing temperature. The characteristic exchange bias field $H_{\mathrm{EB}}$ and the coercive field $H_{C}$ are estimated using the relations $H_{\mathrm{EB}}=$ $\left(H_{C+}+H_{C-}\right) / 2$ and $H_{C}=\left(H_{C+}-H_{C-}\right) / 2$, where $H_{C+}$ and $H_{C-}$ are coercive fields for the positive and negative field axes of the original $M(H)$ loop, respectively as mentioned earlier. Figures 3(j) and 3(k) summarize the temperature de-
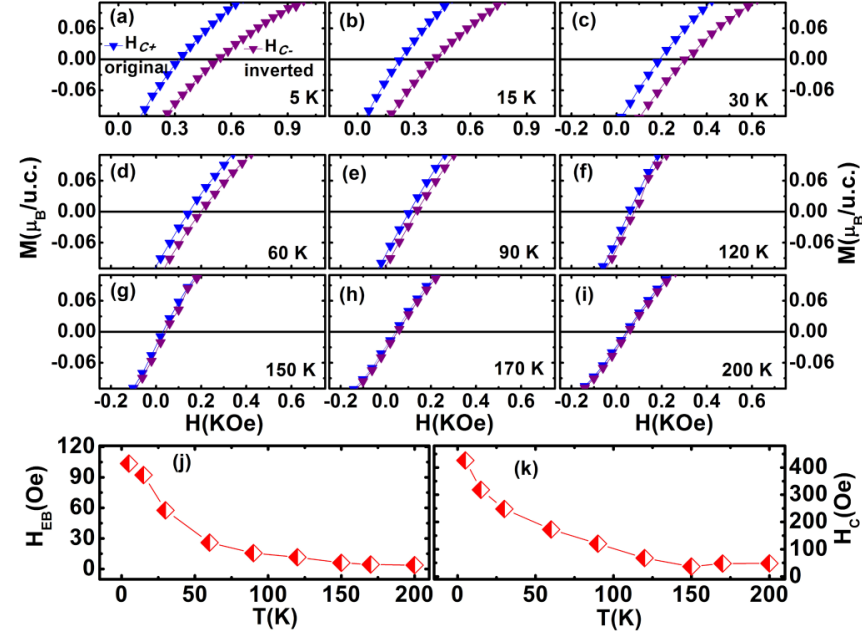

FIG. 3. Temperature dependence of magnetic exchange bias effect: (a)-(i) enlarged view of the original and inverted $M(H)$ loops of the $\left[\operatorname{SCO}_{\mathrm{PC}}(\sim 24 \mathrm{~nm}) / \operatorname{SCO}_{\mathrm{BM}}(\sim 2 \mathrm{~nm})\right]$ bilayer. (j), (k) The estimated $H_{\mathrm{EB}}$ and $H_{C}$ as a function of temperature, respectively.

pendence of $H_{\mathrm{EB}}$ and $H_{C}$ in which a maximum $H_{\mathrm{EB}}$ and $H_{C}$ of 105 and 450 Oe, respectively, is observed at $5 \mathrm{~K}$. Remarkably, it has to be noted that while $H_{\mathrm{EB}}$ gradually falls to zero at $T_{\mathrm{C}} \sim 175 \mathrm{~K}$, a striking nonzero coercive field $H_{C}$ persists beyond $T_{\mathrm{C}}$. Earlier studies on $\mathrm{FM} / \mathrm{AF}(\mathrm{FeNiB} / \mathrm{CoO})$ bilayer with $T_{\mathrm{N}}(291 \mathrm{~K})>T_{\mathrm{C}}(\sim 150 \mathrm{~K})$ reported the persistence of $H_{\mathrm{EB}}$ well above $T_{\mathrm{C}}$, i.e., into the paramagnetic state of the ferromagnet although $H_{C}$ fell to zero at $T_{\mathrm{C}}$ [16]. The induced $H_{\mathrm{EB}}$ in the paramagnetic state in the case of $\mathrm{FeNiB} / \mathrm{CoO}$ bilayer was attributed to modest magnetization in the paramagnetic state of $\mathrm{FeNiB}$, originated either from field-cooling effect or some local ordering at the interface due to close proximity to the $\mathrm{AF}$ layer. Unlike the $\mathrm{FeNiB} / \mathrm{CoO}$ bilayer case, in the present study we do not see any signature MEBE effect, indicating that no net interfacial exchange bias persists above $T_{\mathrm{C}}$. However, the observation of a finite $H_{\mathrm{C}}$ for $T>T_{\mathrm{C}}$ is intriguing. We conjecture that this counterintuitive observation results from the exchange coupling of the magnetic moments in the FM layer to those in the adjacent AF layer, the latter in this case providing an effective staggered external field for the FM spins. The magnetic moments in the AF layer are effectively quenched since $T \ll T_{\mathrm{N}}$. Preliminary Monte Carlo simulations of simple Ising-like models of the coupled FM-AF layers with $T_{\mathrm{C}}<T \ll T_{\mathrm{N}}$ support this hypothesis (see Fig. S12).

Finally, we examine the nature and origin of MEBE in $\left(\mathrm{SCO}_{\mathrm{PC}} / \mathrm{SCO}_{\mathrm{BM}}\right)$ bilayer by performing field-cooled $\mathrm{MHL}$ measurements at $5 \mathrm{~K}$ under varying biasing-field strength as shown in Fig. 4. It is evident that the asymmetry in MHL widens as the biasing cooling-field strength increases. The estimated $H_{\mathrm{EB}}$ under various cooling-field strength shows a saturating tendency towards higher field (a maximum $H_{\mathrm{EB}}$ of $140 \mathrm{Oe}$ is observed at biasing field strength of $5 \mathrm{~T}$ ). Such saturating tendency of $H_{\mathrm{EB}}$ at higher biasing field is common in FM/AF coupled systems unlike the case for coupled FM/SG system in which $H_{\mathrm{EB}}$ typically gets reduced for large cooling field [17].This indicates that the observed interfacial coupling in epitaxial $\left(\mathrm{SCO}_{\mathrm{PC}} / \mathrm{SCO}_{\mathrm{BM}}\right)$ bilayer is $\mathrm{FM} / \mathrm{AF}$ type rather 


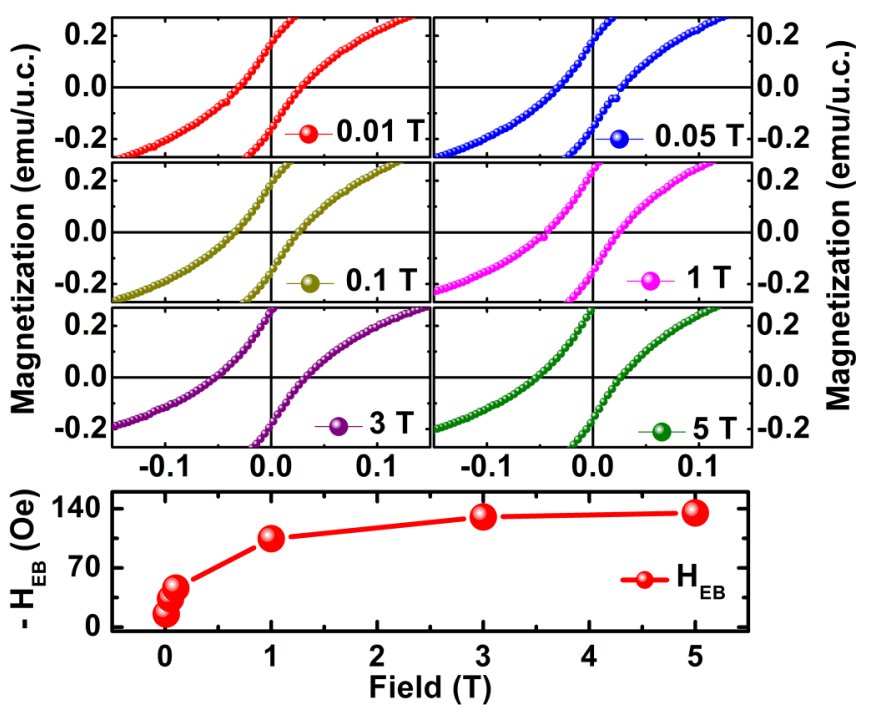

FIG. 4. $M(H)$ loops at $5 \mathrm{~K}$ after field cooling (at various fields) from $350 \mathrm{~K}$ (top panel). $H_{\mathrm{EB}}$ as a function of field (bottom panel).

than FM/SG one. The observations made both from varying biasing-field and time-dependent magnetization study validate each other.

\section{CONCLUSIONS}

In summary, we have demonstrated that a heterointerface involving perovskite-ferromagnet $\mathrm{SrCoO}_{3-\delta}$ and brownmillerite-antiferromagnet $\mathrm{SrCoO}_{2.5}$ with $T_{\mathrm{N}}>T_{\mathrm{C}}$ is formed spontaneously using pulsed-laser epitaxy and exhibits unusual MEBE. This is contrary to common perception in which the $T_{\mathrm{C}}>T_{\mathrm{N}}$ criterion is generally considered to ob- serve MEBE at the FM/AF interface. Structural findings testify to the occurrence of $\mathrm{SrCoO}_{3-\delta} / \mathrm{SrCoO}_{2.5}$ epitaxial natural bilayer on STO. Detailed magnetometry investigations reveal the central footprints for FM/AF interfacial exchange coupling. The possibility of coupled FM/SG interfacial coupling giving rise to $\mathrm{MEBE}$ is ruled out by time-dependent thermoremnant and biasing-field-dependent $H_{\mathrm{EB}}$ measurements. Interestingly, we observe a finite coercive field in the paramagnetic state of $\mathrm{SrCoO}_{3-\delta}$ whereas the exchange bias field vanishes at $T_{\mathrm{C}}$. We conjecture that this counterintuitive observation is due to the effective external quenched staggered field provided by the AF spins. In essence, the present work offers a perspective to design innovative interfaces between oxides with different structural symmetry and explore emergent interfacial phenomena. Moreover, we believe that the observation of MEBE in $\left(\mathrm{SCO}_{\mathrm{PC}} / \mathrm{SCO}_{\mathrm{BM}}\right)$ bilayer will extend the realm of exchange bias beyond conventional systems and broaden the search to a greater combination of FM/AF bilayers with $T_{\mathrm{N}}>T_{\mathrm{C}}$ resulting in such effect. The basic understanding of such unusual exchange bias phenomena will trigger a big step forward in thin film and interfacial magnetism.

\section{ACKNOWLEDGMENTS}

We are grateful to Sachin Sarangi for his superb technical support during magnetic measurements. We thank Gopal Pradhan for fruitful discussion. We thank Zhicheng Zhong for reading the manuscript and for suggestions. We thank T. Som for extending laboratory facility. D.S. and B.C.B. acknowledge the financial support from Max-Planck Society through Max Planck Partner Group. S.G.B. acknowledges the INSPIRE Faculty Fellowship Programme (DSTO1899) for the financial support.
[1] F. Hellman, A. Hoffmann, Y. Tserkovnyak, G. S. D. Beach, E. E. Fullerton, C. Leighton, A. H. MacDonald, D. C. Ralph, D. A. Arena, H. A. Dürr, P. Fischer, J. Grollier, J. P. Heremans, T. Jungwirth, A. V. Kimel, B. Koopmans, I. N. Krivorotov, S. J. May, A. K. Petford-Long, J. M. Rondinelli, N. Samarth, I. K. Schuller, A. N. Slavin, M. D. Stiles, O. Tchernyshyov, A. Thiaville, and B. L. Zink, Rev. Mod. Phys. 89, 025006 (2017).

[2] J. Noguésa, J. Sorta, V. Langlaisb, V. Skumryeva, S. Suriñachb, J. S. Muñozb, and M. D. Baró, Phys. Rep. 422, 65 (2005).

[3] M. Bibes, J. E. Villegas, and A. Barthelemy, Adv. Phys. 60, 5 (2011).

[4] C. Chappert, A. Fert, and F. N. Van Dau, Nat. Mater. 6, 813 (2007).

[5] W. H. Meiklejohn and C. P. Bean, Phys. Rev. 102, 1413 (1956).

[6] W. H. Meiklejohn and C. P. Bean, Phys. Rev. 105, 904 (1957).

[7] J. Nogues and I. K. Schuller, J. Magn. Magn. Mater. 192, 203 (1999).

[8] M. Kiwi, J. Magn. Magn. Mater. 234, 584 (2001).

[9] J. F. Ding, O. I. Lebedev, S. Turner, Y. F. Tian, W. J. Hu, J. W. Seo, C. Panagopoulos, W. Prellier, G. Van Tendeloo, and T. Wu, Phys. Rev. B. 87, 054428 (2013).

[10] T. Yu, X. K. Ning, W. Liu, J. N. Feng, X. G. Zhao, and Z. D. Zhang, J. Appl. Phys. 116, 083908 (2014).
[11] Y. K. Liu, Y. W. Yin, S. N. Dong, S. W. Yang, T. Jiang, and X. G. Li, Appl. Phys. Lett. 104, 043507 (2014).

[12] Y. F. Tian, O. I. Lebedev, V. V. Roddatis, W. N. Lin, J. F. Ding, S. J. Hu, S. S. Yan, and T. Wu, Appl. Phys. Lett. 104, 152404 (2014).

[13] D. Mauri, H. C. Siegmann, P. S. Bagus, and E. Kay, J. Appl. Phys. 62, 3047 (1987).

[14] N. C. Koon, Phys. Rev. Lett. 78, 4865 (1997).

[15] X. W. Wu and C. L. Chien, Phys. Rev. Lett. 81, 2795 (1998).

[16] J. W. Cai, K. Liu, and C. L. Chien, Phys. Rev. B. 60, 72 (1999).

[17] G. S. Alvarez, J. Sort, S. Surinach, M. D. Baro, and J. Nogués, J. Am. Chem. Soc. 129, 9102 (2007).

[18] Y. Long, Y. Kaneko, S. Ishiwata, Y. Taguchi, and Y. Tokura, J. Phys.: Condens. Matter 23, 245601 (2011).

[19] C. K. Xie, Y. F. Nie, B. O. Wells, J. I. Budnick, W. A. Hines, and B. Dabrowsk, Appl. Phys. Lett. 99, 052503 (2011).

[20] T. Takeda, Y. Yamaguchi, and H. Watanabe, J. Phys. Soc. Jpn. 33, 970 (1972).

[21] A. Muñoz, C. de la Calle, J. A. Alonso, P. M. Botta, V. Pardo, D. Baldomir, and J. Rivas, Phys. Rev. B 78, 054404 (2008).

[22] H. Jeen, W. S. Choi, M. D. Biegalski, C. M. Folkman, I. C. Tung, D. D. Fong, J. W. Freeland, D. Shin, H. Ohta, M. F. Chisholm, and H. N. Lee, Nat. Mater. 12, 1057 (2013). 
[23] H. Jeen, W. S. Choi, J. W. Freeland, H. Ohta, C. U. Jung, and H. N. Lee, Adv. Mater. 25, 3651 (2013).

[24] W. S. Choi, H. Jeen, J. H. Lee, S. S. Ambrose Seo, V. R. Cooper, K. M. Rabe, and H. N. Lee, Phys. Rev. Lett. 111, 097401 (2013).

[25] N. Lu, P. Zhang, Q. Zhang, R. Qiao, Q. He, H.-B. Li, Y. Wang, J. Guo, D. Zhang, Z. Duan, Z. Li, M. Wang, S. Yang, M. Yan, E. Arenholz, S. Zhou, W. Yang, L. Gu, C.-W. Nan, J. Wu, Y. Tokura, and P. Yu, Nature (London) 546, 124 (2017).

[26] B. Cui, C. Song, F. Li, X. Y. Zhong, Z. C. Wang, P. Werner, Y. D. Gu, H. Q. Wu, M. S. Saleem, S. S. P. Parkin, and F. Pan, Phys. Rev. Appl. 8, 044007 (2017).

[27] J. Zhang, Z. Zhong, X. Guan, X. Shen, J. Zhang, F. Han, H. Zhang, H. Zhang, X. Yan, Q. Zhang, L. Gu, F. Hu, R. Yu, B. Shen, and J. Sun, Nat. Commun. 9, 1923 (2018).

[28] T. L. Meyer, H. Jeen, X. Gao, J. R. Petrie, M. F. Chisholm, and H. N. Lee, Adv. Electron. Mater. 2, 1500201 (2016).

[29] See Supplemental Material at http://link.aps.org/supplemental/ 10.1103/PhysRevB.99.024425 for details of thin-film growth, structural characterization, substrate background correction from the magnetic data and related magnetization plots, and the results from Monte Carlo simulations.

[30] H. Jeen and H. N. Lee, AIP Adv. 5, 127123 (2015).
[31] M. E. Fisher and M. N. Barber, Phys. Rev. Lett. 28, 1516 (1972).

[32] C. Xiea, J. I. Budnick, and B. O. Wells, Appl. Phys. Lett. 91, 172509 (2007).

[33] J. R. Petrie, C. Mitra, H. Jeen, W. S. Choi, T. L. Meyer, F. A. Reboredo, J. W. Freeland, G. Eres, and H. N. Lee, Adv. Funct. Mater. 26, 1564 (2016).

[34] H. Taguchi, M. Shimada, and M. Koizumi, J. Solid State Chem. 29, 221 (1979).

[35] S. J. Callori, S. Hu, J. Bertinshaw, Z. J. Yue, S. Danilkin, X. L. Wang, V. Nagarajan, F. Klose, J. Seidel, and C. Ulrich, Phys. Rev. B 91, 140405(R) (2015).

[36] A. Migliorini, B. Kuerbanjiang, T. Huminiuc, D. Kepaptsoglou, M. Muñoz, J. L.F. Cuñado, J. Camarero, C. Aroca, G. VallejoFernández, V. K. Lazarov, and J. L. Prieto, Nat. Mater. 17, 28 (2018).

[37] M. Ali, P. Adie, C. H. Marrows, D. Greig, B. J. Hickey, and R. L. Stamps, Nat. Mater. 6, 70 (2007).

[38] J. A. Mydosh, Spin Glasses: An Experimental Introduction (Taylor \& Francis, London, 1993).

[39] Y. Zhou, L. Miao, P. Wang, F. F. Zhu, W. X. Jiang, S. W. Jiang, Y. Zhang, B. Lei, X. H. Chen, H. F. Ding, H. Zheng, W. T. Zhang, J.-f. Jia, D. Qian, and D. Wu1, Phys. Rev. Lett. 120, 097001 (2018). 